\title{
A simulation study for the effect of diffractive collisions on the air shower developments
}

\author{
Ken Ohashi $^{* a}$, Hiroaki Menjo ${ }^{a}$, Yoshitaka Itow ${ }^{a b}$, Takashi Sako $^{c}$, and Katsuaki \\ Kasahara $^{d}$ \\ ${ }^{a}$ Institute for Space-Earth Environmental Research, Nagoya University, Nagoya, Japan \\ ${ }^{b}$ Kobayashi-Maskawa Institute, Nagoya University, Nagoya, Japan \\ ${ }^{c}$ Institute for Cosmic Ray Research, the University of Tokyo, Kashiwa, Japan \\ ${ }^{d}$ Shibaura Institute of Technology, Tokyo, Japan \\ E-mail: ohashi.kendisee.nagoya-u.ac.jp
}

\begin{abstract}
The mass composition of ultra-high energy cosmic rays is important to understand their origin. The maximum depth of air shower developments, $X_{\max }$, is one of the indicators of the mass composition. However, the prediction of $X_{\max }$ depends on the choice of the hadronic interaction model, which makes it difficult to interpret the mass composition. Diffractive collision is a collision type of hadronic interactions and one of the proposed sources of this uncertainty. In this study, we estimate the effect of the fraction of the diffractive collision on the prediction of the mean of $X_{\max }$ for the $10^{19} \mathrm{eV}$ proton incident case by using air shower simulation package CONEX 5.64 and by artificially modifying the fraction of the diffractive collisions in the air shower simulation. The effect of the fraction difference among the major interaction models is estimated to be $8.9 \mathrm{~g} / \mathrm{cm}^{2}$, which is non-negligible. Furthermore, we demonstrate that even if the same fraction of diffractive collisions is used in the models, the discrepancy between the current models in the $X_{\max }$ prediction does not reduce. Other sources of model discrepancy such as particle production after diffractive collisions must be studied more carefully.
\end{abstract}

36th International Cosmic Ray Conference -ICRC2019-

July 24th - August 1st, 2019

Madison, WI, U.S.A.

\footnotetext{
* Speaker.
} 


\section{Introduction}

To understand the origin of ultra-high energy cosmic rays, it is important to measure mass composition. The maximum depth of the air shower developments, $X_{\max }$, is an indicator of the mass composition used in ultra-high energy cosmic-ray observations. Heavier nuclei, such as iron nuclei, show a smaller value of the mean of $X_{\max }$ than lighter nuclei. Therefore, we can discriminate, for example, cosmic-ray protons and irons by comparing $X_{\max }$ observed by ultra-high energy cosmic-ray experiments and the $X_{\max }$ predictions by a Monte Carlo (MC) simulations. However, discrepancies in $X_{\max }$ predictions due to the choice of hadronic interaction models make it difficult to interpret the mass compositions from observations [四]. The source of the uncertainty of mass composition caused by hadronic interaction models needs to be elucidated for precisely understanding the mass composition.

Diffractive collision is a collision type of hadronic interactions and one of the proposed sources of this uncertainty. Diffractive collisions have four types, projectile single-diffractive collisions (projectile SD), target single-diffractive collisions (target SD), double-diffractive collisions (DD), and central-diffractive collisions (CD). Projectile SD is the collision type at which a projectile cosmic ray dissociates, whereas the target air nucleus does not change. In target SD, a target air nucleus dissociates, whereas the projectile cosmic ray does not change. In DD, both target air nucleus and projectile cosmic ray dissociate, and in $\mathrm{CD}$, both are scattered, but some particles are produced. In this study, inelastic collisions other than diffractive collisions are called nondiffractive collisions (ND). Different types of collision have different effects on $X_{\max }$ predictions. If the collision type of the first interaction in the air shower is target SD or CD, for example, $X_{\max }$ predictions are one interaction length larger than that of the ND case because the projectile cosmic ray does not change in that collision. For projectile SD and DD cases, the number of produced particles in the collision is smaller and the energy of produced particles is larger than the ND case, which leads to larger $X_{\max }$ predictions.

The effects of diffractive collisions on the air shower developments were discussed in previous studies [0, 3]; however, the realistic effects of the detailed characteristics of diffractive collisions, such as the fraction of diffractive collision types, on air shower developments are not well understood yet. In this study, we estimate the effect of the fraction of diffractive collision types on the predictions of the mean of $X_{\max },\left\langle X_{\max }\right\rangle$, by using the air shower simulation package CONEX 5.64 [四]. In Section 3, we discuss in detail the fraction of collision types and the relations between collision type and $\left\langle X_{\max }>\right.$ predictions to understand which parameter affects $\left\langle X_{\max }>\right.$ predictions. The effects of diffractive collisions are estimated in Section 4, and we provide conclusions in Section 5.

\section{Simulation setup}

In this work, we use the air shower simulation package CONEX 5.64 [四]. For studying the diffractive collisions in air showers, we modified CONEX in two ways: first, CONEX output was modified to provide the collision type at the first interaction in the air shower. Second, resampling by using collision types was added to modify the ratio among collision types for the whole air shower. The details of the latter modification are described in Section 4.2. The primary particles 
are $10^{19} \mathrm{eV}$ protons, and the zenith angle of these particles is $60^{\circ}$. For each of the three highenergy hadronic interaction models, EPOS-LHC [[]], QGSJET II-04 [四], and SIBYLL 2.3c [0, []], 40,000 events are generated. UrQMD v1.3.1 [9] is used for low-energy hadronic interactions below $80 \mathrm{GeV}$. For the definition of collision types, we use information of each hadronic interaction model. This definition is the same as in a previous study [B]].

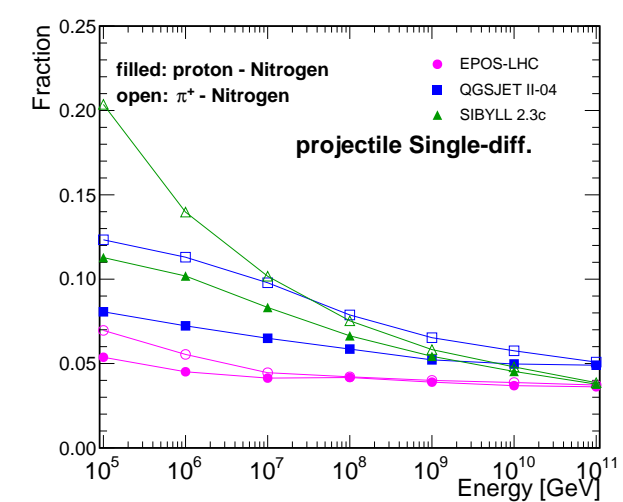

(a) projectile $\mathrm{SD}$

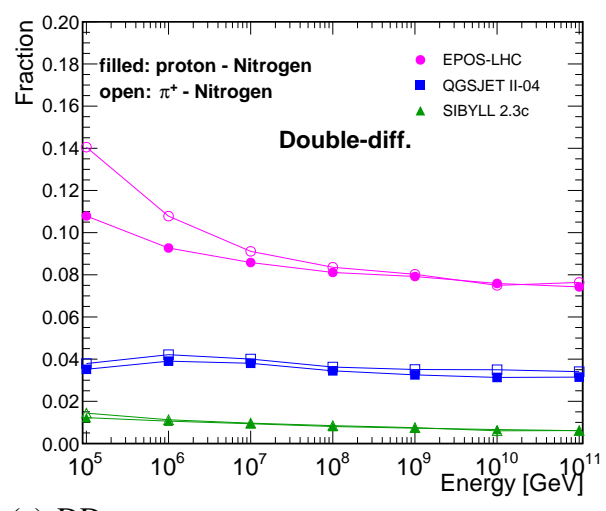

(c) DD

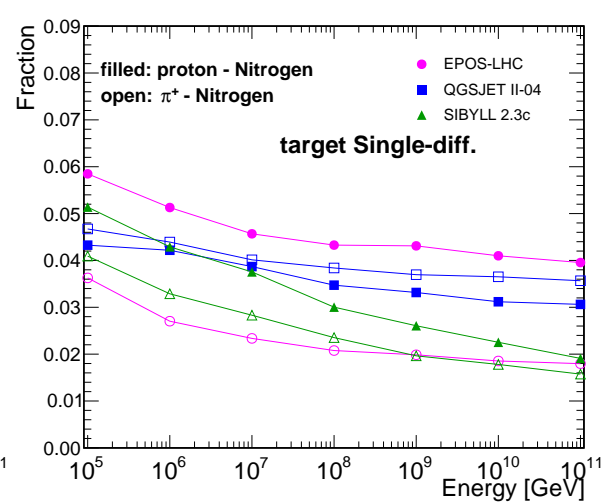

(b) target SD

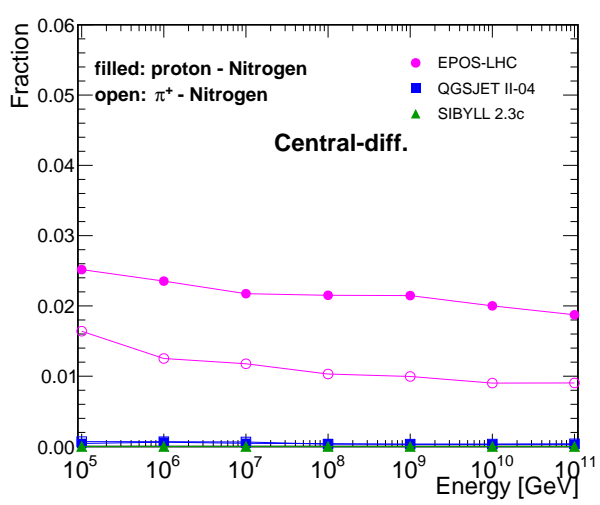

(d) $\mathrm{CD}$

Figure 1: The fraction of collision types: (a) projectile SD, (b) target SD, (c) DD, and (d) CD simulated by using CRMC v1.7 [0]]

\section{The fraction of collision types and the relation to $X_{\max }$}

Before estimating the effect of diffractive collisions, we focus on the fraction of the collision types to understand which parameter affects $X_{\max }$ predictions. First, we check the fraction of the collision types for proton-Nitrogen and $\pi^{+}$-Nitrogen collisions over a wide incident energy range using the event generator interface CRMC v1.7 [ए]]. Figure प shows the results of the fraction, which show the large model discrepancies in the fractions of diffractive collision types. These discrepancies can be the source of the model discrepancies of $X_{\max }$ predictions. As shown in Fig. [ (a)(b), the fractions of the projectile SD and target SD depend on the incident energy, the incident particle, i.e. proton or $\pi^{+}$, and the interaction model. In contrast, as seen in Fig. W(c), dependence in the incident particles is small in DD. The fraction of CD is very small in QGSJET II-04 and SIBYLL 2.3c, as seen in Fig. $⿴ 囗$ (d). 
Next, to understand the relation between collision type and $X_{\max }$ prediction, we categorize the events by the collision type of the first interaction in the air shower and calculate $\left\langle X_{\max }\right\rangle$ predictions for each category. This categorization using information of the first interaction allows us to understand the effect of collision types in the simplest way. Figure $\square$ (a) shows the result of $<X_{\max }>$ of each category. $\left\langle X_{\max }\right\rangle$ predictions of categories of diffractive collisions are larger than those of ND, and that of target SD is the largest among the categories. These results mean that the following two factors affect the model uncertainties of $<X_{\max }>$ predictions:

- The different modeling of particle production in the diffractive collisions, and

- The difference of the fraction of collision types.

Figure $\square$ (b) shows the differences of $\left\langle X_{\max }\right\rangle$ among the categories. The differences between diffractive collisions and ND are 30 to $50 \mathrm{~g} / \mathrm{cm}^{2}$, while those between DD and projectile SD are almost zero consistently. These results mean that the ratio of the fraction of projectile SD to DD does not affect the $\left\langle X_{\max }\right\rangle$ predictions, while other ratios, such as the ratio of the fraction of diffractive collisions to ND or target SD to total SD, affect $\left.<X_{\max }\right\rangle$ predictions because of the differences between $<X_{\max }>$ predictions among the categories. Figure $\square$ (c) shows the differences of $\left\langle X_{\max }\right\rangle$ among the models. The discrepancies among models are almost the same between $\mathrm{ND}$ and target SD. This is because a projectile proton does not change in target SD, so $\left\langle X_{\max }\right\rangle$ becomes one interaction length larger than ND. The discrepancies in $\left\langle X_{\max }\right\rangle$ predictions of ND and target SD among the models are larger than those of projectile SD and DD. The different modeling of diffractive collisions affects the discrepancies in the projectile SD and DD cases, while that of non-diffractive collisions affects ND and target SD cases, so these results mean that the effect of different modeling of diffractive collisions is smaller than that of non-diffractive collisions.

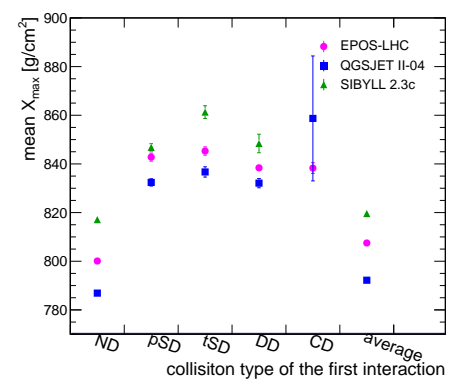

(a) the predictions of mean $X_{\max }$

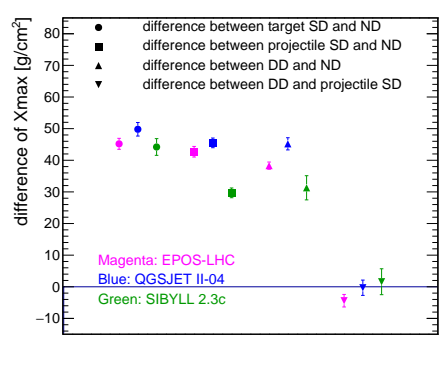

(b) Differences of mean $X_{\max }$ among collision types

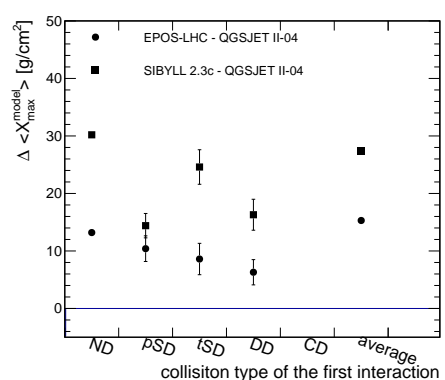

(c) Differences of mean $X_{\max }$ among models

Figure 2: The prediction of mean $X_{\max }$ of each category for three hadronic interaction models (a) and its differences among categories (b) and among models (c). The differences among models $\left.\Delta<X_{\max }^{\text {model }}\right\rangle$ is mean $X_{\max }$ of EPOS-LHC or SIBYLL 2.3c subtracted by that of QGSJET II-04. The horizontal axes of (a) and (c) denote the collision type of the first interaction and the average of the collision types. Here, pSD means projectile SD and $\mathrm{SD}$ means target SD.

In this study, we focus on the effect of differences of the fraction of collision types among the models. Because the discrepancies of $\left\langle X_{\max }\right\rangle$ predictions of projectile SD and DD among the models are not so large, the effect of different modellings of diffractive collisions is not expected 
to be very large, while the effect of the fraction is not well understood yet. Therefore, we estimate the effect of the fraction quantitatively in the next section.

\section{The effect of the fraction of diffractive collisions on $<X_{\max }>$}

\subsection{The effect of diffractive collision at the first interaction on $\left\langle X_{\max }\right\rangle$}

To estimate the effect of the fraction at the first interaction in the air shower, we changed the ratios of the collision type at the first interaction within the range of the model-to-model variation. The cross-section fraction of each collision type to the total inelastic cross section is defined as $f$. To vary the $\mathrm{f}$ values, we introduced the following four parameters $R_{i} . R_{1}$ is the ratio of all diffractive collisions to the total inelastic collisions, $R_{2}$ is the ratio of SD collisions to sum of SD and $\mathrm{DD}$, and $R_{3}$ is the ratio of target $\mathrm{SD}$ to $\mathrm{SD} . R_{4}$ is the ratio of $\mathrm{CD}$ to all diffractive collisions. $f$ can be calculated from these four ratios as shown in Equations 4.1 ,

$$
\begin{array}{r}
f^{N D}=1-R_{1}, \\
f^{\text {projectile } S D}=R_{1}\left(1-R_{4}\right) R_{2}\left(1-R_{3}\right), \\
f^{\text {target } S D=R_{1}\left(1-R_{4}\right) R_{2} R_{3},} \\
f^{D D}=R_{1}\left(1-R_{4}\right)\left(1-R_{2}\right), \\
f^{C D}=R_{1} R_{4} .
\end{array}
$$

The predictions of these ratios are different among different models. For the $R_{1}$ case, the predictions are $0.074,0.11$, and 0.18 by SIBYLL 2.3c, QGSJET II-04, and EPOS-LHC, respectively, so the range of the predictions among models is $0.074-0.18$. In this analysis, the range of the predictions among models is used as the realistic range of ratios. To estimate the effect of model discrepancies of these ratios, we changed the ratio within the range of predictions. Since one of the models does not have CD, we do not change $R_{4}$.

$<X_{\max }>$ after modification of the ratio, $\left.<X_{\max }^{\operatorname{modified}}\right\rangle$, is calculated by using the following equation:

$$
<X_{\max }^{\text {modified }}>=\sum_{i} f^{i}<X_{\max }^{i}>
$$

where $i$ is one of the categories of $\mathrm{ND}$, projectile $\mathrm{SD}$, target $\mathrm{SD}, \mathrm{DD}$, and $\mathrm{CD} .<X_{\max }^{i}>$ is the $<X_{\max }>$ prediction of the category $i$, and $f^{i}$ is the fraction of the category $i$ after modification of one of the ratios. The results of $\left\langle X_{\max }^{\text {modified }}\right\rangle$ are shown in Figure []. When $R_{1}$ is shifted to the value of EPOS-LHC, $\left\langle X_{\max }^{\operatorname{modi}}{ }^{2}\right\rangle$ increases by $3.2 \mathrm{~g} / \mathrm{cm}^{2}$ from the original $\left\langle X_{\max }\right\rangle$ of QGSJET II-04. This is a $3.7 \mathrm{~g} / \mathrm{cm}^{2}$ increase in the case of SIBYLL $2.3 \mathrm{c}$.

Finally, the maximum size of the effect for the ratio is estimated by using Equation 4.3]:

$$
E_{\max }^{R_{j}}=\sum_{i} f_{R_{j}}^{i} \text { Max. }<X_{\max }^{i}>-\sum_{i} f_{R_{j}}^{i} \operatorname{Min} .<X_{\max }^{i}>
$$

where $f_{R_{j} \text { Max. }}^{i}\left(f_{R_{j} \text { Min. }}^{i}\right)$ is the fraction when $R_{j}$ is the maximum (minimum) within the range of predictions. The maximum effect $E_{\max }^{R_{1}}$ is $5.05 \mathrm{~g} / \mathrm{cm}^{2}$, which is $18.4 \%$ of the current size of the model discrepancy of $\left\langle X_{\max }>\right.$ predictions and is non-negligible. $E_{\max }^{R_{2}}$ and $E_{\max }^{R_{3}}$ are $0.37 \mathrm{~g} / \mathrm{cm}^{2}$ and $0.19 \mathrm{~g} / \mathrm{cm}^{2}$, respectively, which is smaller than $1.5 \%$ of the current model discrepancy and 
is negligible. These analyses only consider the collision at the first interaction, so these results are underestimated. We extend the analysis to all hadronic collisions in the air shower in the next section.

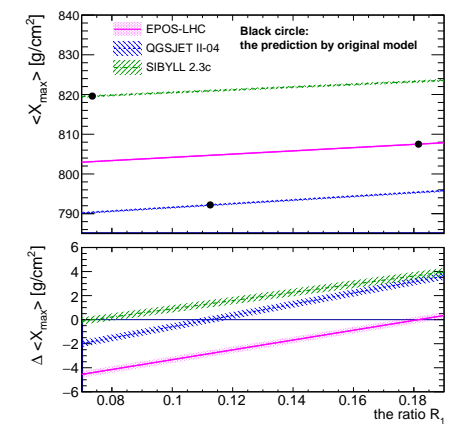

(a) the ratio $R_{1}$

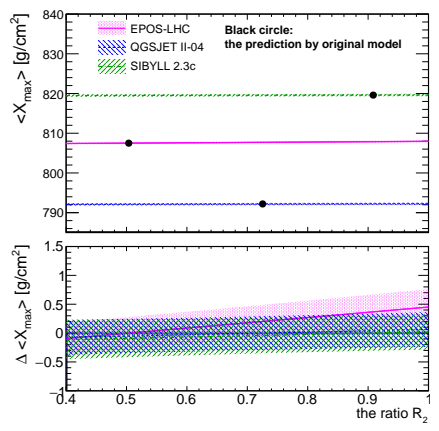

(b) the ratio $R_{2}$

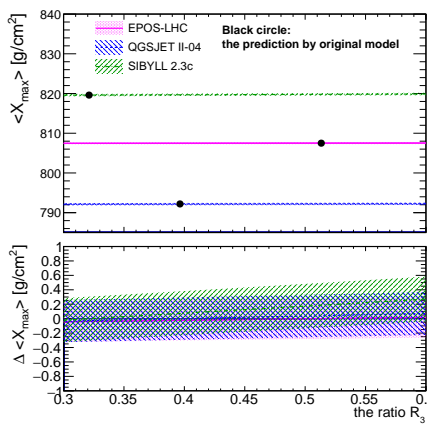

(c) the ratio $R_{3}$

Figure 3: The ratio dependence of $<X_{\max }>$ for (a) the ratio $R_{1}$, (b) $R_{2}$, and (c) $R_{3}$ for EPOS-LHC (solid line), QGSJET II-04 (dashed line), and SIBYLL 2.3c (dash-dotted line). Hatched regions show statistical errors. The upper panel of each graph shows the $\left\langle X_{\max }>\right.$ as a function of the ratio, and black circles show the predictions of the original model. The bottom panel shows $\left.\Delta<X_{\max }\right\rangle$, which is $\left\langle X_{\max }^{\text {original }}\right\rangle-<$ $X_{\max }^{\text {modified }}>$, where $<X_{\max }^{\text {original }}>$ is the prediction of the original model and $\left\langle X_{\max }^{\text {modified }}\right\rangle$ is the modified prediction. The energy of a primary proton is $10^{19} \mathrm{eV}$.

\subsection{The effect of the fraction of diffractive collisions in the air shower}

To consider the effect of the ratio $R_{1}$ for all hadronic interactions in the air shower, we artificially changed the ratio of collision types by resampling the collision in the simulation. The procedure of resampling is as follows:

1. The ratio after resampling (the target ratio) is defined using the ratio simulated by CRMC.

2. When a hadronic collision with the concerning collision type is generated in the air shower, the output collision type is chosen randomly by following the target ratio. A collision is regenerated until a collision with the chosen collision type is generated.

3. Resampling is performed for all interactions above $10^{15} \mathrm{eV}$.

Figure $₫$ shows the ratio $R_{1}$ in the proton-Nitrogen and $\pi^{+}$-Nitrogen collisions simulated by CRMC. Energy and the incidence particle dependence of the ratio $R_{1}$ are shown. The target of the ratio is defined by fitting predictions of EPOS-LHC to the equation $a \log _{10}(E)+b$ above $10^{15} \mathrm{eV}$. The target of the baryon incident collisions is a function fitted to proton-Nitrogen collisions, and that of the meson incident collisions is a function fitted to $\pi^{+}$-Nitrogen collisions.

The air shower events with this resampling are simulated using CONEX 5.64. The number of events is 40,000 . Table $\square$ shows the results of resampling of $R_{1}$. The targets of the ratio are taken from EPOS-LHC, so the systematic uncertainty is estimated by comparing $<X_{\max }>$ predictions of the original simulation using EPOS-LHC and that after resampling using EPOS-LHC. The size of the effect of the resampling is $8.9 \mathrm{~g} / \mathrm{cm}^{2}$ for SIBYLL $2.3 \mathrm{c}$ and $4.4 \mathrm{~g} / \mathrm{cm}^{2}$ for QGSJET II-04, which 


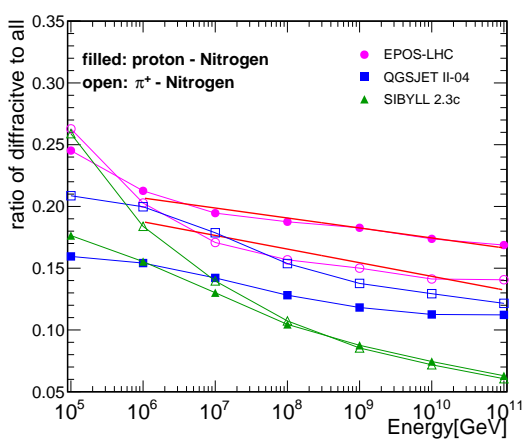

Figure 4: The ratio of diffractive collisions to all collisions $\left(R_{1}\right)$ simulated by using CRMC v1.7 [प]d]. The ratio $R_{1}$ of EPOS-LHC is fitted by the equation $a \log _{10}(E)+b$ above $10^{15} \mathrm{eV}$ (straight solid line).

Table 1: The effect of the ratio of diffractive collisions to all collisions in the air shower. The target of resampling is set by fitting the ratio of predictions by EPOSLHC with energy above $10^{15} \mathrm{eV}$. The unit is $\mathrm{g} / \mathrm{cm}^{2}$.

\begin{tabular}{l|ccc}
\hline & \multicolumn{3}{|c}{$X_{\max }\left[\mathrm{g} / \mathrm{cm}^{2}\right]$} \\
model & model original & with resampling & difference \\
\hline EPOS-LHC & $807.5 \pm 0.3$ & $809.5 \pm 0.3$ & $2.0 \pm 0.4$ \\
QGSJET II-04 & $792.2 \pm 0.3$ & $796.6 \pm 0.3$ & $4.4 \pm 0.4$ \\
SIBYLL 2.3c & $819.6 \pm 0.3$ & $828.5 \pm 0.3$ & $8.9 \pm 0.4$ \\
\hline model discrepancy & 27.4 & 31.9
\end{tabular}

is $32.5 \%$ and $16.1 \%$ of the current size of the model discrepancy of $\left\langle X_{\max }\right\rangle$ predictions. The systematic uncertainty is $2.0 \mathrm{~g} / \mathrm{cm}^{2}$, and the statistical uncertainty is $0.4 \mathrm{~g} / \mathrm{cm}^{2}$. These results mean that the effect of the ratio of $R_{1}$ is non-negligible. As discussed in the previous section, $\left\langle X_{\max }\right\rangle$ becomes larger by 3.2 to $3.7 \mathrm{~g} / \mathrm{cm}^{2}$ when $R_{1}$ at the first interaction is shifted to the value of EPOSLHC; therefore, the effect of changing fractions in the whole air shower is 1 to 2 times as large as that at the first interaction in the air shower. This feature indicates that the fraction of the first interaction is very important, and the fractions of the secondary interactions have non-negligible effects.

Table $\mathbb{W}$ denotes important information. The model discrepancy of the $\left\langle X_{\max }\right\rangle$ prediction between EPOS-LHC and QGSJET II-04 decreases by $2.4 \mathrm{~g} / \mathrm{cm}^{2}$ after resampling, but that between EPOS-LHC and SIBYLL $2.3 \mathrm{c}$ increases by $6.9 \mathrm{~g} / \mathrm{cm}^{2}$. Therefore, the model discrepancies of $<X_{\max }>$ predictions among the three models increase by $4.5 \mathrm{~g} / \mathrm{cm}^{2}$ after resampling. These results mean that the ratio $R_{1}$ has a non-negligible effect on the $\left\langle X_{\max }>\right.$ prediction; however, if the ratio $R_{1}$ of the three models is set to the same value after improvements, model discrepancies become larger.

\section{Discussion and conclusion}

In this study, we estimated the effect of diffractive collisions by focusing on the fraction of the collision types. The effect of the ratio of diffractive collisions to all inelastic collisions was $32.5 \%$ of the current size of the model discrepancies of $<X_{\max }>$ predictions among models, which was 
non-negligible. The effects of the ratios of the fraction among the diffractive collision types, for example, the ratio of SD to SD and DD, were smaller than $1.5 \%$ of the current model discrepancy and were negligible. However, the $\left\langle X_{\max }\right\rangle$ after resampling showed larger model discrepancies than the current one. These results mean that the effect of the fraction of diffractive collisions is one of the sources of model discrepancies, but not the main source of the current model discrepancy. Therefore, we need to consider other sources of the model discrepancy.

The model discrepancies of the fraction of projectile SD and DD were large, and these were the main sources of discrepancies of the ratio $R_{1}$ among models. These model discrepancies in the fraction of projectile SD and DD were also large at $10^{17} \mathrm{eV}$, which corresponds to the energy of nucleon-nucleon collisions in the Large Hadron Collider at CERN. It is important to measure these fractions by collider experiments for reducing the uncertainty of $\left\langle X_{\max }\right\rangle$ predictions caused by the fraction of collision type.

\section{Acknowledgements}

We thank N. Sakurai for providing the programs for resampling in CONEX. We are grateful to F. Riehn for useful discussions and comments.

\section{References}

[1] J. Bellido et al., Depth of maximum of air-shower profiles at the Pierre Auger Observatory: Measurements above $10^{17.2} \mathrm{eV}$ and Composition Implications, PoS(ICRC2017) 506.

[2] S.Ostapchenko, LHC data on inelastic diffraction and uncertainties in the predictions for longitudinal extensive air shower development, Physical Review D 89 (2014) 074009.

[3] L. B. Arbeletche, V. P. Goncalves, and M. A.Muller, Investigating the influence of diffractive interactions on ultra - high energy extensive air showers, International Journal of Modern Physics A 33 (26) 01.

[4] T. Bergmann et al., One-dimensional hybrid approach to extensive air shower simulation, Astropart. Phys.26(2007) 420-432.

[5] T. Pierog, I. Karpenko, J. M. Katzy, E. Yatsenko, and K. Werner, EPOS LHC: Test of collective hadronization with data measured at the CERN Large Hadron Collider, Physical Review C 92 (2015) 034906.

[6] S. Ostapchenko, Monte Carlo treatment of hadronic interactions in enhanced Pomeron scheme: I. QGSJET-II model, Physical Review D 83 (2011) 014018.

[7] E.-J. Ahn, R. Engel, T. K. Gaisser, P. Lipari, and T. Stanev, Cosmic ray interaction event generator SIBYLL 2.1, Physical Review D 80 (2009) 094003.

[8] F. Riehn et al., The hadronic interaction model Sibyll 2.3c and Feynman scaling, PoS ICRC2017 (2017) 301.

[9] S.A. Bass et al., Prog.Part.Nucl.Phys. 41 (1998) 225; M. Bleicher et al., J. Phys. G25 (1999) 1859.

[10] T. Pierog, C. Baus, R. Ulrich, https://web.ikp.kit.edu/rulrich/crmc.html 